\title{
Handover Management in LEO Satellite Network using Area and Angle Based Algorithm
}

\author{
Debabrata Sarddar ${ }^{1}$, *Priyajit Sen $^{2}$ and Himadri Biswas ${ }^{3}$ \\ ${ }^{1}$ Assistant professor, Department of Computer Science and Engineering, \\ University of Kalyani \\ ${ }^{* 2}$ Student of Master of Technology, Department of Computer Science and \\ Engineering, University of Kalyani \\ ${ }^{3}$ Assistant Professor, Department of Computer Applications, Bengal College of \\ Engineering \& Technology, Durgapur, West Bengal, INDIA \\ dsarddar1@gmail.com, priyajit91@gmail.com,mr.himadri.biswas@gmail.com
}

\begin{abstract}
LEO satellites are nearer to the earth's surface than that of the other satellite systems. $L E O$ satellites revolve round the earth is in the range from $500-1500 \mathrm{~km}$ from the surface of the earth. The satellite is placed closer to the surface of the earth. If the height of the satellite is increased by increasing the power of the equipment used in the satellite system, the beam of the satellite cover a wide area in the surface of the earth. The radius of the foot print will increase as the height will increase and as the radius will increase the area of coverage will also increase. Height $(h)$ is directly proportional to the radius $(r)$ and area $(A)$ is directly proportional to the square of the radius. Thus, the number of satellite required to cover the whole earth will decrease. The handoff failure will be decreased.
\end{abstract}

Keywords: Handoff, LEO, Debu's Constant, Tangential Angle, Degradation Interval.

\section{Introduction}

\section{Satellite Systems}

Satellites are far away from the surface of the earth. They can cover a wider area on the surface of the earth. The information to be transmitted from a mobile user (MS) must be correctly received by a satellite and forwarded to one of the earth stations (ESs). Line of Sight communication between the mobile user and the satellite is possible.

\section{Types of Satellite Systems}

1. GEO (Geostationary Earth Orbit) satellites are about $36,000 \mathrm{~km}$ above the earth's surface.

2. LEO (Low Earth Orbit) satellites are about $500-1500 \mathrm{~km}$ above the earth's surface.

3. MEO (medium earth orbit) or ICO (intermediate circular orbit) are about 6000 $20,000 \mathrm{~km}$ above the earth's surface.

4. HEO (Highly Elliptical Orbit) having an elliptical orbit. 
Satellite orbiting path is shown in the figure below:

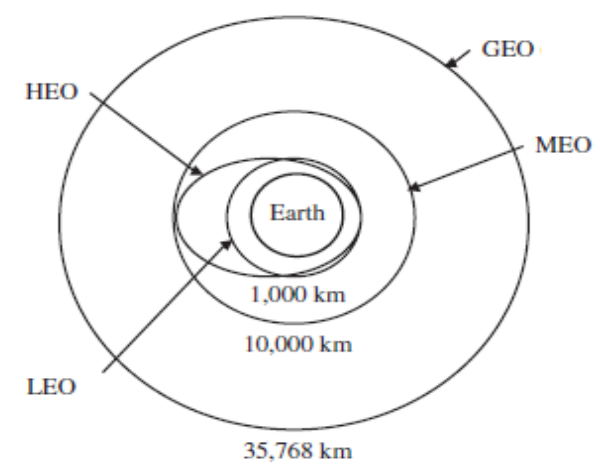

Figure 1. Orbit of Different Satellites

\section{According to Newton's gravitational law:}

Attractive Force $\left(F_{g}\right)=m g(R / r)^{2}$

Centrifugal Force $\left(F_{c}\right)=m r \omega^{2}$

Angular velocity of the Satellite $(\omega)=2 \Pi f_{r}$

\section{Assumptions:}

1. $m$ is the mass of the satellite,

2. $g$ is the gravitational acceleration of the earth $(g=9.81 \mathrm{~m} / \mathrm{s} 2)$,

3. $R$ is the radius of the earth $(\mathrm{R}=6370 \mathrm{~km})$,

4. $r$ is the distance of the satellite from the center of the earth,

5. $\omega$ is the angular velocity of the satellite, and

6. $f$ is the rotational frequency.

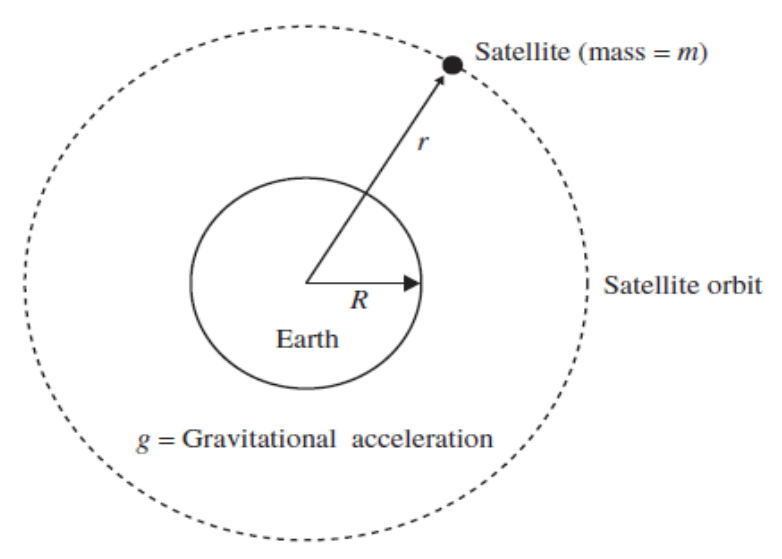

Figure 2. Earth Satellite Parameters for a Stable Orbiting Path

Thus,

$\frac{F_{g}}{F_{c}}=\frac{m g(R / r)^{2}}{m r \omega^{2}}$ 


$$
\begin{aligned}
& =\frac{m g(R / r)^{2}}{m r\left(2 \pi f_{r}\right)^{2}} \\
& =\frac{g R^{2}}{r^{3}\left(2 \pi f_{r}\right)^{2}}
\end{aligned}
$$

Therefore, $r=\sqrt[3]{\frac{g R^{2}}{\left(2 \pi f_{r}\right)^{2}}}, r$ is the distance from the satellite from the center of the earth.

\section{Footprint:}

The footprint of a satellite is the ground area that offers coverage in the surface of the earth.

\section{Spot beam:}

The coverage area of every satellite is divided into slightly overlapping cells, which are called spot beams.

\section{Base Station:}

The base station keeps track of all Mobile Stations within its coverage range, controls the allocation and de-allocation of radio channels and performs the intelligent and decision making process to reduce the computational effort and the weight of the satellites.

\section{Handoff:}

Whenever an Mobile Station(MS) moves from one satellite coverage area to an area served by any other satellite, the MS needs to be connected with the new satellite via Base Station(BS) by rejecting the connection of old satellite.

a) Intra-satellite handover: It occurs when movement of the Mobile Station (MS) occurs from one spot-beam to another spot-beam in the same footprint of the satellite due to its relative motion with respect to the satellite.

b) Inter-satellite handover: It occurs when the Mobile Station (MS) leaves the footprint of the present satellite and come into the footprint of another satellite.

c) Gateway handover: It occurs when gateway leaves the footprint and the mobile station (MS) remains in the footprint of the satellite.

d) Inter system handover: It occurs from the satellite network to a terrestrial cellular network which is cheaper and have lower latency.

\section{Related Work}

A work is done on LEO satellite network to manage handoffs by measuring the distance and angle of the satellite from earth's surface [1]. Blocking a handoff call is generally considered less desirable from user's point of view than blocking a new call request since dropping a call in progress breaches quality of service (QOS) requirements [2]. Quality of service handover management is done over LEO/MEO satellite systems [3]. A research work is done over handover queuing strategies with dynamic and fixed channel allocation techniques in LEO mobile satellite systems [4]. Another work is done over Handover and channel assignment in Mobile Cellular Networks [5]. Elastic handover scheme was proposed by the author of paper [6] for the LEO satellite mobile communication systems. 


\section{Proposed Approach}

LEO satellites are generally placed within the range of $500 \mathrm{~km}$ to $1500 \mathrm{~km}$ from the surface of the earth. As the height will increase, the beam of satellite will cover a wider area of the earth as the radius is increasing while the beam is coming to the earth by making tangential angle with the surface of the earth. So considering the earth as a circle, the area of the footprint will be $A=\pi r^{2}, r$ is the radius of the footprint. As the height of the satellite is increasing the radius will also increase and as the radius will increase the area will also increase. Thus a wide area can be covered by the beam of the satellite. The handoff will occur less than that of the satellite belong to the closer to the surface of the earth. Thus by increasing the height of the satellite from the surface of the earth, it will also increase the area of coverage. Thus the number of satellite will also decrease to cover the globe. The cost may increase to place the satellite at a distant location; cost due to number of required satellite to cover the whole earth will decrease. So a large number of people will get the coverage of the satellite for a long time. Therefore,

$\mathrm{A} \propto \mathrm{r}^{2}$

$\mathrm{A} \infty \mathrm{h}$

By Join variation,

$\mathrm{A} \infty \mathrm{r}^{2} \mathrm{~h}$

Therefore,

$A=D r^{2} h$, where $D$ is proportionality Constant

$\mathrm{D}$ is called Debu's Constant.

Now, consider the threshold angle is $\infty_{\mathrm{t}}$, crossing which handoff will occur. It will be considered just before that place where the degradation (back and forth between two spot beams frequently) occurs. After crossing the threshold angle, reaching at the degradation interval, the mobile devices searches for a proper channel belonging to another footprint area of the same or another satellite. An active scan or a passive scan can be used for searching as per the profitability of the channel through which handoff will occur. As in the figure,

Let the height of the satellite from the center of the first footprint is $\mathrm{h}_{1}$ and that of the second footprint is $h_{2}$. Radius of the first footprint and second footprint are $r_{1}, r_{2}$ respectively. Also let that, $\alpha$ and $\beta$ are the threshold angle for first footprint and second footprint respectively.

I) suppose the distance covered by the mobile device on first footprint during degradation interval is $d_{1}$. Thus, the distance from the center of first footprint to the point of threshold angle (wherefrom degradation starts) is $\left(\mathrm{r}_{1}-\mathrm{d}_{1}\right)$. We can find out the value of $\mathrm{r}_{1}$ in the following approach:

We can get the co-ordinate of the satellite and the position where satellite beam makes a tangential angle with the surface of the earth via GPS transmitter. Let the co-ordinate of satellite system is $\left(\mathrm{x}_{1}, \mathrm{y}_{1}\right)$ and co-ordinate of the point where beam of the satellite touches the surface of the earth is $\left(\mathrm{x}_{2}, \mathrm{y}_{2}\right)$. Therefore, the distance is measured as,

Distance $(\mathrm{d})=\sqrt{(y 2-y 1)^{2}+(x 2-x 1)^{2}}$

Therefore, the hypotenuse is the distance measure i.e. $\mathrm{d}$ which is actually $\mathrm{AB}$ for the first footprint.

From Figure 3, $\sin \gamma=\frac{h 1}{d}$

i.e. $\gamma=\sin ^{-1} \frac{h_{1}}{d}$ 
Now, $\mathrm{r} 1=\frac{\hbar 1}{\tan Y}$

For the first footprint as the threshold angle is $\alpha$,

$\tan \alpha=\frac{\text { his }}{r 1-d 1}$

Therefore, $\alpha=\tan ^{-1} \frac{h 1}{r 1-d 1}$

Remember that, $\alpha>\gamma$ and the handoff will occur as the mobile device crosses the threshold angle $\alpha$ during the degradation interval and there is no need of handoff as it crosses $\gamma$.

II) Again, suppose the distance covered by the mobile device on second footprint during degradation interval is $\mathrm{d}_{2}$. Thus, the distance from the center of second footprint to the point of threshold angle (wherefrom degradation starts) is $\left(\mathrm{r}_{2}-\mathrm{d}_{2}\right)$. We can find out the value of $r_{2}$ in the following approach:

Here, the hypotenuse is the distance measure i.e. $\mathrm{d}$ which is actually $\mathrm{CD}$ for the second footprint.

From Figure $3, \sin \delta=\frac{h_{2}}{d}$

i.e. $\delta=\sin ^{-1} \frac{h 2}{d}$

Now, $\mathrm{r}_{2}=\frac{\hbar_{2} 2}{\tan 8}$

Now for the second footprint as the threshold angle is $\beta$,

$\tan \beta=\frac{h 2}{\mathrm{r} 2-\mathrm{d} 2}$

Therefore, $\beta=\tan ^{-1} \frac{h_{2}}{r 2-d 2}$

Remember that, $\beta>\delta$ and the handoff will occur as the mobile device crosses the threshold angle $\beta$ during the degradation interval and there is no need of handoff as it crosses $\delta$.

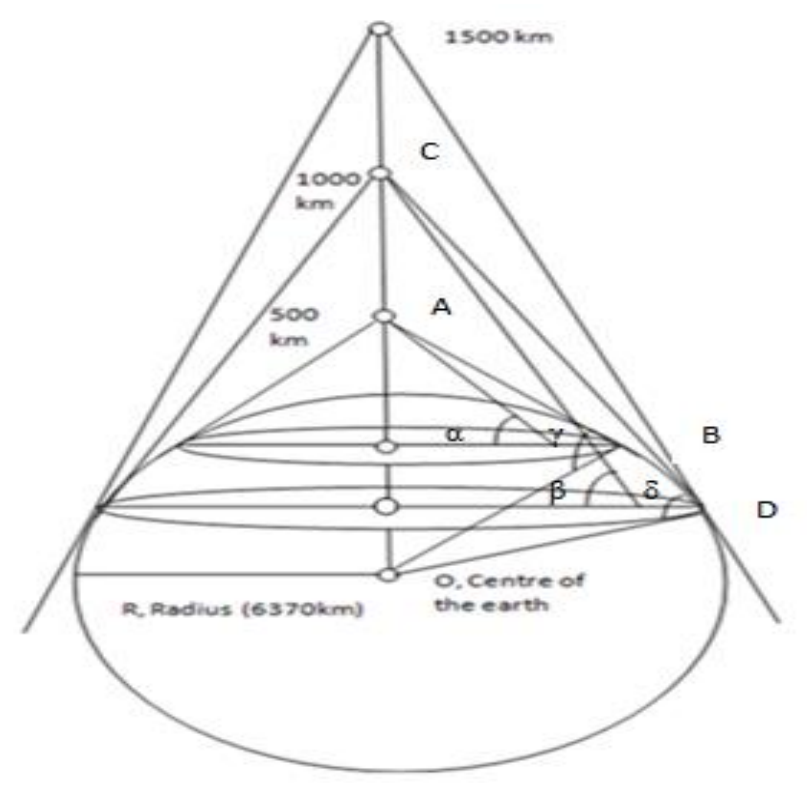

Figure 3. Altitude of the LEO Satellites from the Surface of the Earth 


\section{Algorithm}

\section{A. Area measurement Algorithm:}

1. Start

2. Let $r$ be the radius of the footprint of a satellite, distance of the satellite from the earth surface is $\mathrm{h}$ and $\mathrm{A}$ is the area to be covered by the footprint.

3. The area (A) of the footprint can be calculated in the following approach:

$\mathrm{A} \infty \mathrm{r}^{2}\left[\mathrm{as}, \mathrm{A}=\pi \mathrm{r}^{2}\right]$

$\mathrm{A} \infty \mathrm{h}$

By Join variation,

$\mathrm{A} \infty \mathrm{r}^{2} \mathrm{~h}$

Therefore,

$\mathrm{A}=\mathrm{D} \mathrm{r}^{2} \mathrm{~h}$, where $\mathrm{D}$ is proportionality Constant

$\mathrm{D}$ is called Debu's Constant. The value of $\mathrm{D}$ is nearer to 1 as the height of the satellite increases and radius of the footprint is nearer to the radius of the earth.

4. End

\section{B. Angle Measurement Algorithm:}

1. Start

2. Let the height of the satellite from the center of the first footprint $\left(\mathrm{h}_{1}\right)=500 \mathrm{~km}$. The height of the satellite from the center of the second footprint $\left(\mathrm{h}_{2}\right)=1000 \mathrm{~km}$. Radius of the first footprint is $\mathrm{r} 1$ and second footprint is $\mathrm{r} 2 . \infty$ is the threshold angle for first footprint and $\beta$ is the threshold angle for second footprint.

3. Let the distance covered by the mobile device on first footprint during degradation interval is $d_{1}$ and the same for second footprint is $d_{2}$.Thus, the distance from the center of first footprint to the point of threshold angle (wherefrom degradation starts) is $\left(r_{1}-d_{1}\right)$ and the same for second footprint is $\left(\mathrm{r}_{2}-\mathrm{d}_{2}\right)$.

4. Let the co-ordinate of satellite system is $\left(\mathrm{x}_{1}, \mathrm{y}_{1}\right)$ and co-ordinate of the point where beam of the satellite touches the surface of the earth is $\left(\mathrm{x}_{2}, \mathrm{y}_{2}\right)$.

Distance $(d)=\sqrt{(y 2-y 1)^{2}+(x 2-x 1)^{2}}$

5. For the first footprint, $\sin \gamma=\frac{h 1}{d}$ i.e. $\gamma=\sin ^{-1} \frac{h 1}{d}$

Now, $\mathrm{r} 1=\frac{\hbar \mathrm{n} 1}{\tan Y}$

Let, the threshold angle is $\infty$,

$\tan \alpha=\frac{k 1}{r_{11-d 1}}$

Therefore, $\alpha=\tan ^{-1} \frac{h 1}{r 1-d 1}$

6. For the second footprint, $\sin \delta=\frac{h 2}{d}$ i.e. $\delta=\sin ^{-1} \frac{h_{2}}{d}$

Now, $r_{2}=\frac{\hbar 2}{\tan 8}$

Let the threshold angle is $\beta$, 
$\tan \beta=\frac{\mathrm{h} 2}{\mathrm{r} 2-\mathrm{d} 2}$

Therefore, $\beta=\tan ^{-1} \frac{h 2}{r 2-d 2}$

7. End

\section{Simulation Results}

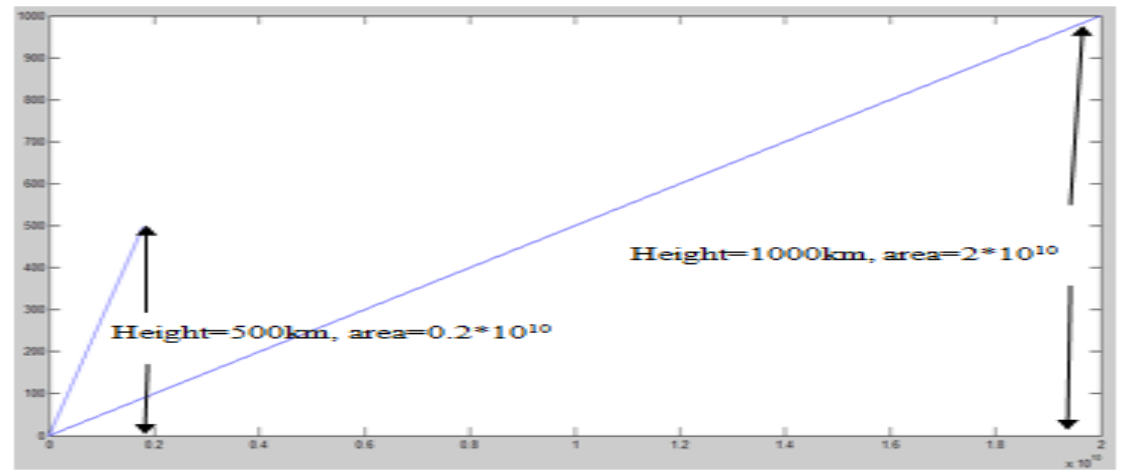

Figure 4. Measurement of Area of Coverage of the Footprint of Satellite with Respect to Height

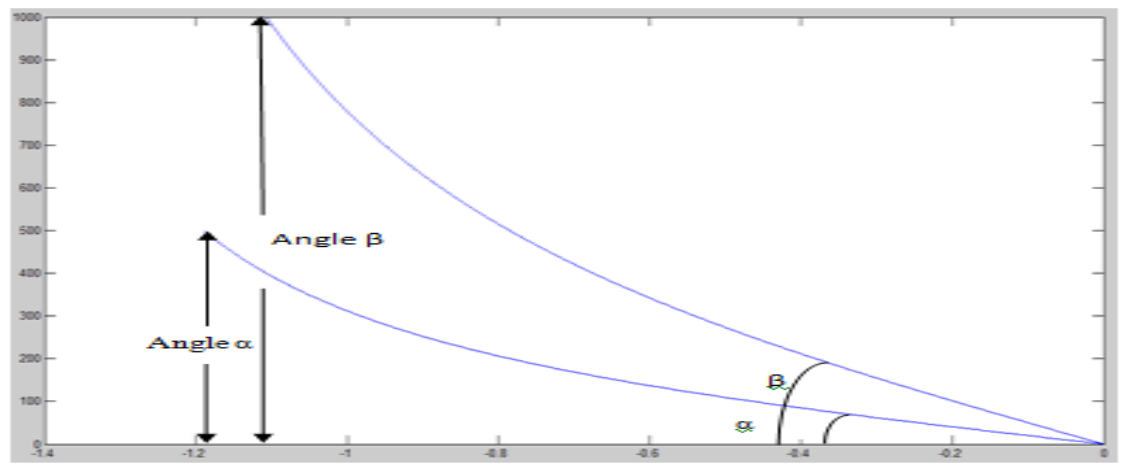

Figure 5. Measurement of Threshold Angle wherefrom Handoff occurs ( $\alpha$ and $\beta$ )

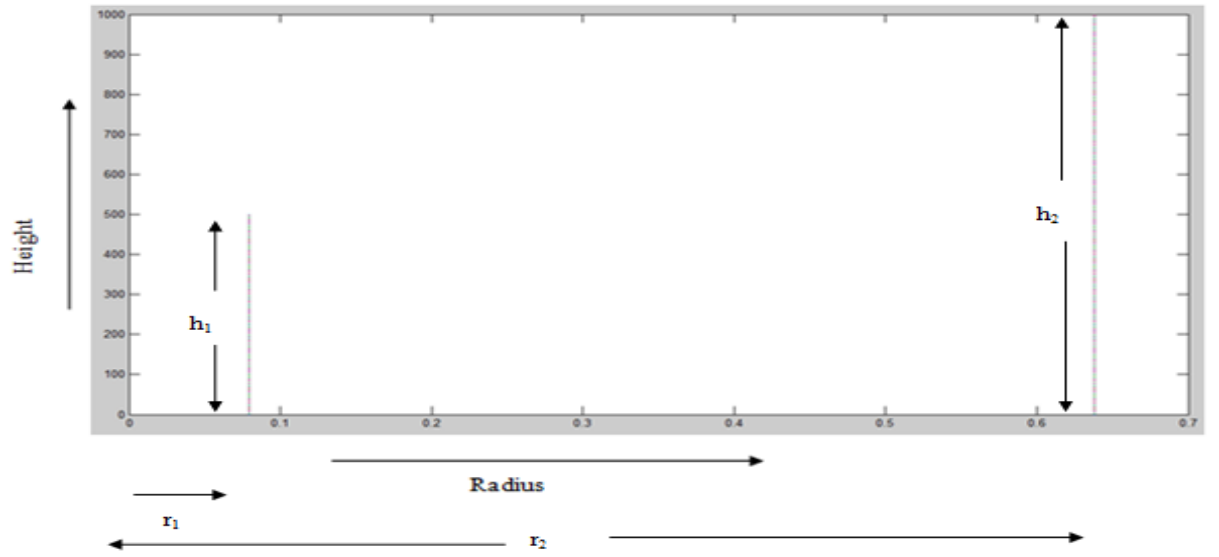

Figure 6. Measurement of Radius with Respect to Height

(When Height is 500 km, Radius is Less than 0.1 and when Height is 1000 $\mathrm{km}$, Radius is Less than 0.7 


\section{Conclusion}

We have seen that, as the height of the satellite increases, the radius also increases. Thus the area is increases. To calculate the distance of the satellite from the point of tangential angle (where satellite beam touches the surface of the earth) gps transmitter will be used to compute the co-ordinate of the two points. Whenever the mobile device crosses threshold angle, the handoff may occur i.e. for the first footprint as the mobile user crosses angle $\alpha$ then handoff may occur during the degradation interval. No handoff will occur as the mobile device crosses $\gamma$. Note that, $\alpha>\gamma$. Handoff will occur only when the proper channel will be found during the degradation interval in the footprint of the same satellite or another. One of the problem is the false handoff may occur during the degradation interval. We can calculate the radius of the both footprint (produced by changing the height of the satellite). Again threshold angle and the tangential angle are calculated as proposed in the algorithm and in the simulation result. Thus, finally as the area increases many mobile devices can stay within the footprint of same satellite thus the chance of handoff will be decreased and less number of satellites will be required to cover the whole earth.

\section{Future Work}

Our future work may be manage the condition whenever mobile devices face the problem of false handoff that is when a mobile device crosses the threshold angle during the degradation interval and reaches the another spot beam leaving current spot beam to check for the proper channel. If proper channel is not found then false handoff will occur. The future intention is to minimize the false handoff.

\section{References}

[1] Debabrata Sarddar, Subhajit Chatterjee, Sougata Chakraborty, Pradipta Ghosh, Kunal Hui, Mrinal Kanti Naskar, "A handover management in LEO satellite Network using Angular and Distance Based Algorithm", International Journal of Computer Applications(0975-8887), Volume 31- No. 5, October 2011.

[2] Papapetrou, E. and Pavlidou, F.-N.,(2005) "QOS handover management in LEO/MEO satellite systems", Wireless Personal Communications, vol.24, no.2,pp.189-204, January 2002; Special Issue on Broadband Mobile Terrestrial-Satellite Integrated Systems.

[3] Re, E. Fantacci, D., R., and Giambene, G.,(1999)"Handover queuing strategies with dynamic and fixed channel allocation techniques in low earth orbit mobile satellite systems," IEEE Transactions on Communications, 47(1):89-102, January 1999.

[4] Tekinay, S. and Jabbari, B.,(1991) "Handover and Channel Assignment in Mobile Cellular Networks," IEEE Comm. Mag., vol. 29, no. 11, pp. 42-46, November 1991

[5] Xu, Y., Ding, Q., and Ko, C., (2000) "Elastic Handover scheme for leo satellite mobile communication systems" in IEEE Global Telecomm. Conf., San Francisco, CA, pp. 1161-1165.

[6] http://member.openmobilealliance.org/ftp/public_documents/POC/.

[7] Y.-B. Lin and I. Chlamtac,Wireless and Mobile Network Architecture, John Wiley and Sons, Inc. Hoboken, NJ, 2001.

[8] J. Wang, Q-A. Zeng, and D. P. Agrawal, "Performance Analysis of Preemptive Handoff Scheme for Integrated Wireless Mobile Networks," Proceedings of the IEEE Globecom 2001, November 2001.

[9] Karapantazis,S., Todorova, P., and Pavlidou, F.-N.,(2006) "On bandwidth and inter-satellite handover management in multimedia leo satellite systems," in Proc. Of the Advanced Satellite Mobile Systems (ASMS) Conference 2006, Herrsching am Ammersee, Germany.

[10] Maral, G., Restrepo, J., Re, E. del, Fantacci, R., and Giambene, G., (1998) "Performance analysis for a guaranteed handover service in an LEO constellation with satellite fixed cell," IEEE Trans. Vej. Technol., vol.47, no.4, pp. 1200-1214.

[11] P. K. Chowdhury, M. Atiquzzaman, W. Ivancic, "Handover Schemes in Satellite Networks: State of the art and future research directions" IEEE communications surveys 4TH Quarter 2006, Vol. 8, NO. 4A.

[12] Debabrata Sarddar, Shubhajeet Chatterjee, Ramesh Jana, Shaik Sahil Babu, Hari Narayan Khan, Utpal Biswas and M.K.Naskar, -"Fast Handoff Implementation by using Curve Fitting Equation With Help of GPS|", International Journal of Computer Science issues (IJCSI) Vol. 08, Issue 03, pp. 535-542, No. 1, May 2011, ISSN (Online): 1694-0814. 
[13] Debabrata Sarddar, Soumya Das, Dipsikha Ganguli, Kalyan Kumar Das, Sougata Chakraborty, Kunal Hui and Mrinal Kanti Naskar. Article: A New Method for Controlling Mobility Management Cost of PatHO-LEO satellite and Mobile IP Network. International Journal of Computer Applications 37(7):3238, January 2012. Published by Foundation of Computer Science, New York, USA.

[14] Debabrata Sarddar, Soumya Das, Dipsikha Ganguli, Sougata Chakraborty, Kunal Hui, Kalyan Kumar Das and Mrinal Kanti Naskar. Article: A New Method for Fast and Low Cost Handover in Leo Satellites. International Journal of Computer Applications 37(7):39-45, January 2012. Published by Foundation of Computer Science, New York, USA

[15] E. Cayirci and I. F. Akyildiz, - User mobility pattern scheme for location update and paging in wireless systems, \|I IEEE Trans. Mobile Computing, vol. 1, no. 3, pp. 236-247, 2002.
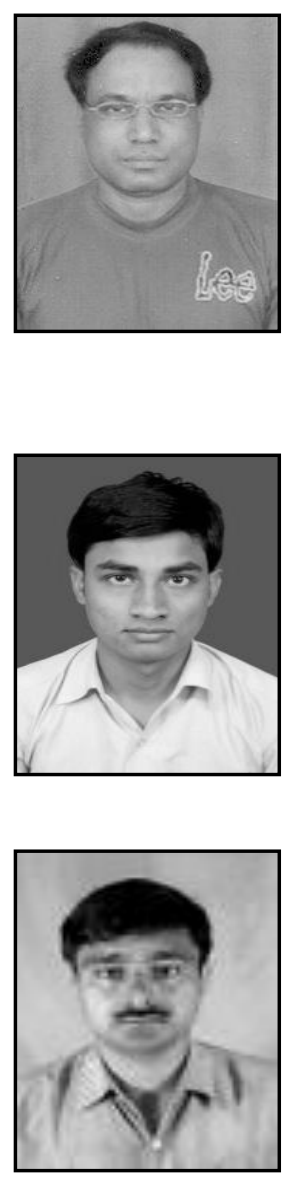

\section{Authors}

Debabrata Sarddar, Assistant Professor in the Department of Computer Science and Engineering, University of Kalyani, Kalyani, Nadia, West Bengal, India. He has done $\mathrm{PhD}$ from Jadavpur University. He completed his M.Tech in Computer Science \& Engineering from DAVV, Indore in 2006, and his B.E in Computer Science \& Engineering from NIT, Durgapur in 2001. He has published more than 150 research papers in different journals and conferences. His research interest includes Mobile Computing, Wireless Sensor Network and Cloud Computing.

Priyajit Sen, $\mathrm{He}$ is presently pursuing M.Tech in Computer Science and Engineering at the Department of Computer Science and Engineering, University of Kalyani, Kalyani, Nadia, West Bengal, India. He completed his MCA from Department of Computer Science and Engineering, University of Kalyani, Kalyani, Nadia, West Bengal, India in 2015. His research interest includes Mobile Computing, Wireless Sensor Network and Cloud Computing.

Himadri Biswas, $\mathrm{He}$ is an Assistant Professor in the Department of Computer Applications, Bengal College of Engineering \& Technology, Durgapur, West Bengal, INDIA. He has also 13 years of teaching experiences and worked under several institutions. He is currently pursuing $\mathrm{PhD}$ from University of Kalyani. He has completed his M. Tech in Computer Science \& Engineering from WBUT in 2010, and his MCA from St. Xavier's College, Kolkata under IGNOU, in 2004. His research interest includes cloud computing, mobile computing 
International Journal of Grid and Distributed Computing

Vol. 9, No. 8 (2016) 